\title{
A Study on the Effect of Neighboring Protons in Proton-Coupled Spin-Lattice Relaxation of Methylene Carbon-13 in $n$-Undecane
}

\author{
Chul Kim and Jo Woong Lee* \\ School of Chemistry, College of Natural Sciences, Seoul National Linersity, Seoul 151-742, Korea \\ Received Januarv $1+, 2002$
}

\begin{abstract}
Proton coupled carbon-13 relaxation experiment was performed to investigate the effect of vicinal protons on spin-lattice relaxation of methylene carbon- 13 in $n$-undecane. A BIRD type pulse sequence was employed as a way to check the validity of describing the ${ }^{13} \mathrm{CH}_{2}$ moiety as an isolated. $1 \mathrm{I}_{2}$ spin system. The results show that the presence of wimal protons exerts substantial influence on the relaxation of methylene carbon- 13 . indicating that it is not a very good approximation to treat a methylene moiety as an isolated. $1 \mathrm{I}_{2}$ spin system.
\end{abstract}

Keywords : Proton-coupled relaxation, $n$-Undecane, Deuteration, BIRD-pulse.

\section{Introduction}

The knowledge of dynanics of methylene moieties is of crucial importance for better understanding of the segmental motions occurring in a long hydrocarbon chain and the study of proton-coupled relaxation of carbon- 13 in $\mathrm{CH}_{2}$ groups can serve usefully for this purpose. ${ }^{1.12}$ In particular, for an $n$ alkane chain molecule. dipole-dipole spectral densities obtained from the proton-coupled carbon-13 relaxation experiments provide us a valuable insight into the segmental motions occurring in the straight carbon backbone.

Grant and coworkers as well as Fuson $e t a l$. utilized the proton-coupled carbon- 13 relaxation as a means of investigating the segmental motions in $n$-nonane labeled at the central carbon. ${ }^{11}$ In order to analyze the observed curves for several relaxation modes they treated the methylene moiety at the central carbon as an isolated $A X_{2}$ spin system and regarded the protons two bonds away from the carbon of interest. which will, for brevity, be henceforth referred to as vicinal protons, merely as a source of randon field. Surprisingly. when theoretical calculations based on the Redfield equation were performed for the $A X_{\Sigma}$ spin sy'stem. this oversimplified view however was found to lead to a good fit with experimental data thus yielding a set of values for various spectral densities. When the vicinal protons were deuterated to minimize the effect originating from them. the theoretical relaxation curves could also be fitted well with the observed ones. but yielding a somewhat different set of values for the dipolar spectral densities. ${ }^{11}$ This means that the assumption that the interaction with vicinal protons may be treated only as a random field term is not very satisfactory and. whenever possible. the deuteration of interfering protons is recommended. Since deuteration of a proton (or protons) at a given carbon site is very laborious and timeconsuning. use of the deuterated sample is not alway's practical and/or feasible.

In the present work we explore a new BIRD-type pulse experiment. besides that employed by Grant et $\alpha f_{.}{ }^{12}$ to

\footnotetext{
"Coresponding author. E-mail: jwleeghnmrlab2.snu.ac.kr
}

tackle this problem. A BIRD pulse is applied to $n$-undecane to invert only those protons directly bonded to the methylene carbon of interest (henceforth referred to as geminal protons). ${ }^{13}$ Relaxation from the initial spin state created in this manner was also observed in addition to those obtained by the conventional Grant method. Simultaneous fitting of these two different types of relaxation curves will obviously give us more leverage to determine the set of values of spectral densities than relying only on the Grant type measurements. Not surprisingly. theoretical calculation based on the $A X_{2}$ model with the effect of vicinal protons being treated as a random field term failed to produce a good simultaneous fit with these two different types of relaxation data. To analyze the situation we assumed that the system may be described as an $A X M$ system. where the geminat protons. $X$, are assumed to be weakly coupled to a vicind proton. $M$, and kept only the dominating terms representing the effect due to the latter protons in the corresponding relaxation matrix. Of course. there are four vicinal protons around a given methylene carbon but we assume their effect may be described by considering only one of them when they are motionally equivalent. This situation will ideally suit for the central carbon site in a hydrocarbon chain. However. if the carbon site of interest is very close to the center of the chain, the same approximation is expected to be equally well valid as we demonstrate in this paper. An $A X M$ model was found to be able to describe both of the two different kinds of experimental data satisfactorily, thus producing a reliable set of parametric values for spectral densities. Hopefully when generalized. we may employ this method as a routine substitute of expensive and painstaking course of deuteration in the study of segmental motions in the chain molecules.

\section{Theory}

Bloch-Redfield theory. The fundamental equation of motion for a nuclear spin system can be derived from the time-dependent Sclurödinger equation. The second-order perturbation theory leads to the so-called Redfield equation 
for the spin density operator: $:^{1+1.15}$

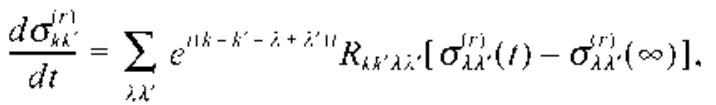

where the elements of the relaxation matrix $\mathbf{R}$ depends on the dipole-dipole and random field spectral densities. When only the longitudinal spin components are relevant, one may use a simpler form of $\mathrm{\Gamma q}$. (1) which can be rewritten in the following form of Solomon equation: ${ }^{16}$

$$
\frac{d N_{i}}{d t}=\sum_{j} W_{u}\left[N_{j}(t)-N_{j}(\infty)\right]
$$

where $N_{1}(t)$ and $N_{i}(\infty)$ are, respectively, the population of the $i$ th spin energy level at time $t$ and at equilibrium and $W_{i j}$ is the rate for transition $j \rightarrow i$. For convenience $\mathrm{Eq}$. (2) may also be written in terms of the symmetrized normal modes as have been shown by Grant ef al., which involves the normalized irreducible tensor operators. Grant et al. have defined a normal magnetization mode as the trace over product of deviation density operator with a corresponding irreducible spherical tensor operator. ${ }^{17-20} \ln$ this formalism each normal magnetization mode can be expressed as a linear combination of the diagonal density-matrix elements and among them as many modes as possible are experimentally measured and compared with theoretically derived expressions.

For our study of a ${ }^{1.5} \mathrm{CH}_{2}$ moiety in $n$-undecane we will briefly outline the application of this formalism to $A X_{2}$ and $A V_{2} M$ spin system. The energy levels and eigenstates for an

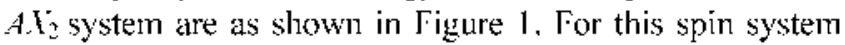
symmetric and antisymmetric normal magnetization modes are expressed as follows:

$$
\begin{aligned}
{ }^{a} v_{1} & =\frac{1}{2}\left(N_{1}-N_{2}+N_{3}+N_{+}-N_{5}-N_{0}-N_{7}-N_{8}\right) \\
& =\left(L_{1}+L_{2}-L_{3}\right) / 2 k_{3} \equiv " v_{+++}
\end{aligned}
$$

$$
\begin{aligned}
& " v_{3}-\frac{1}{\sqrt{2}}\left(N_{1}+N_{2}-N_{7}-N_{8}\right)-\left(L_{3}+L_{\xi}\right) / \sqrt{2} k_{x} \equiv " v_{x} \\
& " v_{\mathrm{j}}=\frac{1}{2}\left(N_{1}-N_{2}-N_{3}-N_{4}-N_{5}-N_{6}+N_{7}-N_{8}\right) \\
& =\left(L_{1}-L_{2}-L_{3}\right) / 2 k_{\lambda} \equiv " v_{--} \\
& " v_{1}=\frac{1}{\sqrt{2}}\left(N_{3}-N_{4}-N_{5}-N_{t}\right) \\
& " v_{5}-\frac{1}{\sqrt{2}}\left(N_{1}-N_{2}-N_{7}+N_{8}\right)-\left(L_{1}-L_{3}\right) / \sqrt{2} k_{4} \\
& -\left(L_{.1}-L_{c}\right) / \sqrt{2} k_{.1} \equiv{ }^{a} v_{1:-} \\
& " v_{0}-\frac{1}{2}\left(N_{1}+N_{2}-N_{3}-N_{3}-N_{5}-N_{0}+N_{7}+N_{8}\right) \\
& " v_{7}=\frac{1}{\sqrt{2}}\left(N_{3}-N_{+}-N_{5}+N_{6}\right) \\
& \text { " } v_{8}=N_{1}+N_{2}+N_{3}-N_{4}-N_{5}+N_{6}+N_{7}+N_{8}=N_{\text {sesterl }} \equiv " v_{r}
\end{aligned}
$$

and the equation of motion for these magnetization modes is

$$
\left.\frac{d v_{i}}{d t}--\sum_{l} \Gamma_{\imath \jmath} \mid v_{i}(t)-v_{i}(\infty)\right)
$$

where the matrix $\Gamma$ is blockdiagonalized into " $\Gamma$ and " $\Gamma$ and their matrix elements are given as below:

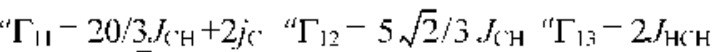

$$
\begin{aligned}
& { }^{4} \Gamma_{\mathrm{I} 4}-7 \sqrt{2} / 3 . J_{\mathrm{HCH}} \\
& " \Gamma_{22}-10 / 3 J_{\mathrm{CH}}-5 J_{\mathrm{HH}}+2 j_{\mathrm{H}} " \Gamma_{23}-2 \sqrt{2} J_{\mathrm{C}} \mathrm{HH} \\
& { }^{\prime \prime} \Gamma_{24}-5 / 3 . J_{\mathrm{HCH}}-2 J_{\mathrm{CHH}} \\
& \text { " } \Gamma_{: 3}-4 J_{(\mathrm{H}}+2 J_{\mathrm{HH}}-2 j_{\mathrm{c}}+4 j_{\mathrm{H}} \\
& \text { " } \Gamma_{34}--\sqrt{2} J_{\mathrm{HCH}}-\sqrt{2} . J_{\mathrm{HH}}-2 \sqrt{2} j_{\mathrm{HH}} \\
& " \Gamma_{44}-14 / 3 J_{{ }^{\prime H} \mathrm{H}}-4 / 3 J_{\mathrm{H}(\mathrm{H}}+J_{\mathrm{HH}}+2 j j_{c}+4 j_{\mathrm{H}}-2 j_{\mathrm{HH}} \\
& { }^{\prime} \Gamma_{11}-16 / 3 J_{\mathrm{CH}}-2 J_{\mathrm{HC}} \mathrm{H}+5 J_{\mathrm{HH}}+2 j_{\mathrm{c}}+2 j_{\mathrm{H}} \\
& { }^{\prime} \Gamma_{12}-5 \sqrt{2} / 3 J_{(\mathrm{H}}+2 \sqrt{2} J_{(\mathrm{HH}} \\
& \Gamma_{1 . \mathrm{i}}-5 / 3 J_{\mathrm{HCH}}-2 J_{\mathrm{C} \mathrm{HH}}
\end{aligned}
$$

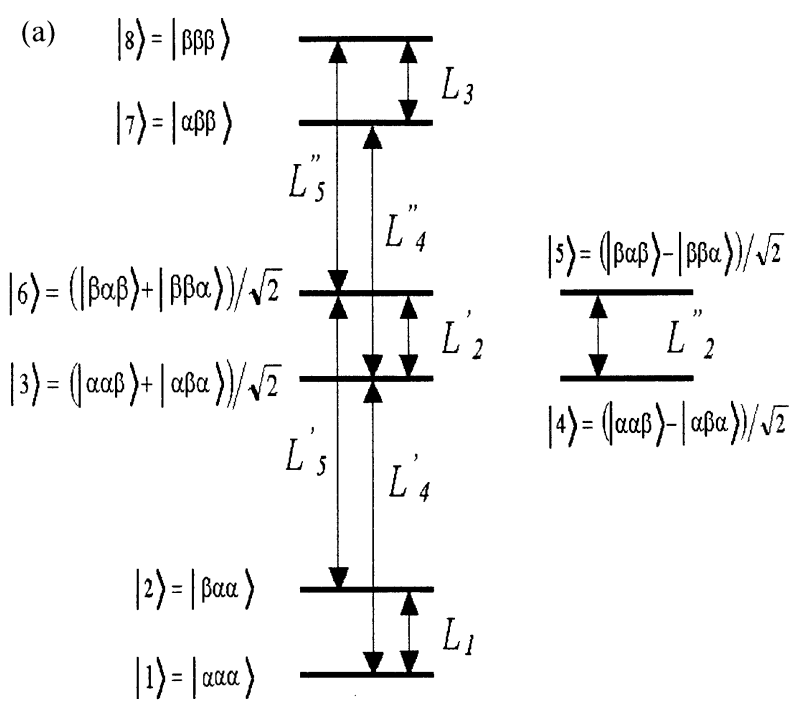

(b)

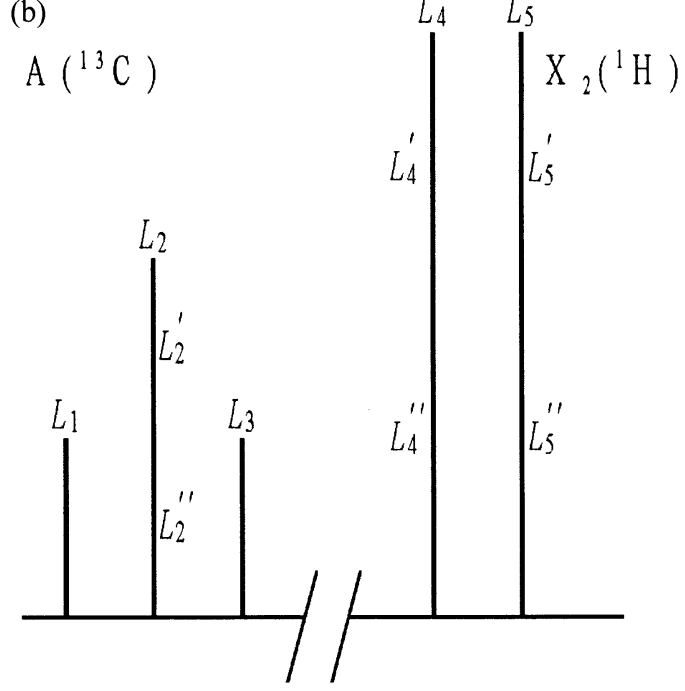

Figure 1. (a) Fnergy level Diagram and Figenstates - spin labels are in the order of C. H. H' spins: L,'s are the allowed (single quantum) transitions that lead to spectral lines shown in (b): (b) $A$ Schematic Spectrum - triplet for $d\left({ }^{13} \mathrm{C}\right.$ ) and doublet for $\lambda_{2}\left({ }^{1} \mathrm{II}\right)$ in isotropic media. 


$$
\begin{aligned}
& { }^{x} \Gamma_{22}=20 / 3 J_{\mathrm{Cl}}+2 J_{\mathrm{HII}}+4 j_{11} \\
& { }^{\mathrm{N}} \Gamma_{2 \xi}=10 \sqrt{2} / 3 J_{\mathrm{ICII}}+\sqrt{2} J_{\mathrm{IEH}}+2 \sqrt{2} j_{\mathrm{III} \text { [ }} \text { and } \\
& { }^{r} \Gamma_{3 \xi}=20 / 3 J_{\mathrm{CII}}-10 / 3 J_{\mathrm{HCII}}+J_{\mathrm{HII}}+4 j_{\mid \mathrm{II}}-2 j_{\text {IIII }}
\end{aligned}
$$

The matrices " $\Gamma$ and $" \Gamma$ are both symmetric, that is, ${ }^{i} \Gamma_{i l}=$ ${ }^{a} \Gamma_{j}$ and ${ }^{s} \Gamma_{i j}={ }^{\prime} \Gamma_{n}$. In isotropic media only four of the modes given in $\Gamma \mathrm{q}$. (3) are directly measurable. They are " $v_{1}$, , the total $A$ magnetization; " $v_{x}$, the total $X$ magnetization: " $v_{1}$, the difference in intensity between the sum of outer two lines and the inner single line of the $A$ triplet, and " $v_{10}$, the difference in intensity between the outer two lines of the $A$ triplet.

For methylene moieties in $n$-undecane the chemical shift differences between geminal and vicinal protons are comparable in magnitude with the scalar spin coupling between them and, therefore, at a first glance we might be tempted to describe these protons as comprising a strongly coupled spin system. However, we can show that the presence of ${ }^{13} \mathrm{C}$ allows us to treat the moiety interacting with a vicinal proton as a weakly coupled $A X_{2} M$ spin system due to large coupling constant between $A$ and $X$ spins where $A$ stands for ${ }^{13} \mathrm{C}$ and $Y$ and $M$, respectively, for geminal and vicinal proton. This means that only the secular term $J_{X M} I_{X:=} I_{1 /=}$, instead of the full interaction $J_{x: 1} \mathbf{I}_{3} \cdot \mathbf{I}_{3,}$, needs to be considered when dealing with the scalar coupling between the two protons. The neglected nonsecular terms can be shown to generate a small amount of coherences of the type $I_{1:} I_{x} I_{1 /}$ and $I_{A z} \Gamma_{1} I_{M}^{+}$ (involving the zero quantum of the protons $X$ and $M$ ) when a BIRD pulse is applied (See Appendix ll). However, this type of coherences was found to quickly decay away (probably due to rapid spin diffusion process of protons) before applying a observing carbon $90^{\circ}$ pulse providing no interference with our measurement. In reality, there are four vicinal protons surrounding a given methylene carbon- 13 atom in $n$ undecane. But their influences on this carbon- 13 atom may be considered approximately additive and can be described in terms of that of a single proton $M$.

The carbon-13 signal for a ${ }^{1} \mathrm{CH}_{2}$ moiety in $n$-undecane basically consists of three lines with the intensity ratio of 1 : $2: 1$ which are separated from each other by the coupling constant ${ }^{3} J_{\mathrm{CH}}(=125 \mathrm{~Hz}$ ). In the presence of vicinal protons each of these lines is split further, but the coupling between the carbon-13 and a vicinal proton is so small that the splitting due to this coupling was hardly resolvable in our case. Therefore, we had to observe the integrated intensities of these unresolved lines in our experiment, which means that we can treat the dynamics of our methylene moiety in terms of various relaxation modes of an $A X_{2}$ system that undergo cross relaxation with the $M$ spin. The energy levels and eigenstates for an $A X_{2} M$ system are as shown in Figure 2.

In dealing with the effect of the vicinal protons, we define symmetric and antisymmetric normal magnetization modes for an $A X_{2} M$ system as follows:

$$
\begin{aligned}
& " v_{1}=\frac{1}{2}\left(N_{1}-N_{2}-N_{3}+N_{4}-N_{5}-N_{6}+N_{7}-N_{8}+N_{9}\right. \\
& \left.-N_{1 j}+N_{11}+N_{12} \quad N_{1, j} \quad N_{1+}+N_{15} \quad N_{1 \omega}\right) \equiv{ }^{a t} v_{111} \\
& " v_{2}-\frac{1}{\sqrt{2}}\left(N_{1}+N_{2}-N_{7}-N_{8}+N_{0}+N_{10}-N_{15}-N_{16}\right) \equiv " v_{3} \\
& " v_{3}-\frac{1}{2}\left(N_{1} \quad N_{2} \quad N_{3} \quad N_{+}\left|N_{5}\right| N_{6}\left|N_{7} \quad N_{\mathrm{s}}\right| N_{3}\right. \\
& \left.-N_{11}-N_{11}-N_{12}+N_{1: 3}+N_{1.1}+N_{15}-N_{10}\right) \equiv " v_{1-1} \\
& " v_{4}=\frac{1}{\sqrt{2}}\left(N_{3}-N_{4}-N_{5}-N_{6}+N_{11}-N_{12}-N_{1.3}-N_{14}\right) \\
& v_{5}-\frac{1}{\sqrt{2}}\left(N_{1} \quad N_{2} \quad N_{7}\left|N_{8} \cdot N_{9} \quad N_{10} \quad N_{15}\right| N_{10}\right) \equiv v_{\cdot n} \\
& v_{0}=\frac{1}{2}\left(N_{1}-N_{2}-N_{3}-N_{4}-N_{5}-N_{6}+N_{7}+N_{8}+N_{9}\right. \\
& \left.-N_{10}-N_{11}-N_{12}-N_{15}-N_{14}+N_{15}+N_{16}\right)
\end{aligned}
$$
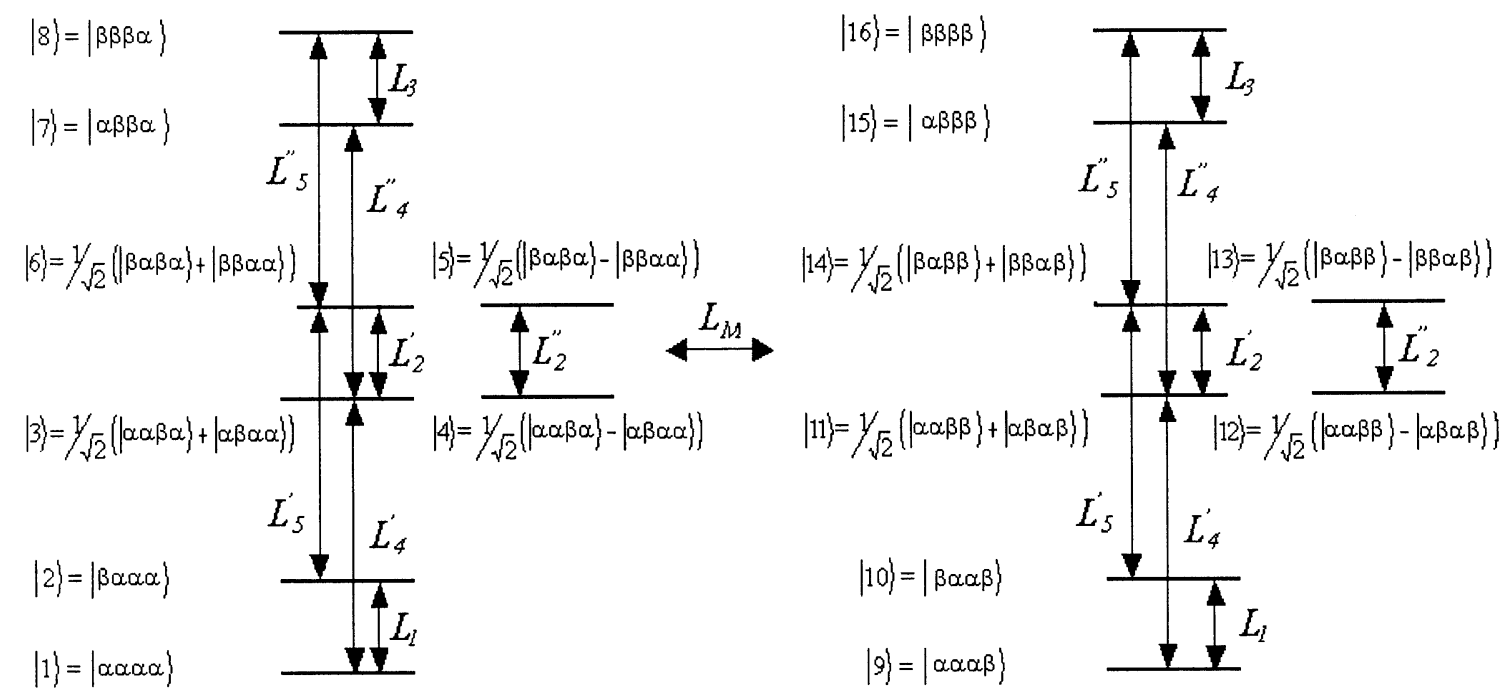

Figure 2. Energy Level Diagram and Eigenstates - spin labels are in the order of C. H. H'. M spins; $L_{\mathrm{r}}$ 's and $L_{15}$ are the allowed (single quantum) transitions of $A d_{2}$ spin and $1 /$ spin respectively. 


$$
\begin{aligned}
& v_{7}-\frac{\mathrm{l}}{\sqrt{2}}\left(N_{s}-N_{1}-N_{5}+N_{0}+N_{11}-N_{1:}-N_{1, j}+N_{1,1}\right) \\
& v_{8}-N_{1}+N_{2}+N_{3}-N_{4}+N_{5}+N_{6}-N_{7}+N_{8}+N_{9}-N_{10} \\
& +N_{11}+N_{12}-N_{13}+N_{14}-N_{15}+N_{16} \equiv v_{t} \\
& v_{9}-\frac{1}{2}\left(N_{1}-N_{2}+N_{3}+N_{4}-N_{5}-N_{6}+N_{7}-N_{8}-N_{9}-N_{10}\right. \\
& \left.-N_{11}-N_{12}+N_{13}+N_{14}-N_{15}+N_{16}\right) \\
& v_{1(11}=\frac{1}{\sqrt{2}}\left(N_{1}+N_{2}-N_{7}-N_{8}-N_{i 5}-N_{1(1}-N_{15}+N_{16}\right) \\
& v_{11}-\frac{1}{2}\left(N_{1}-N_{2}-N_{5}-N_{4}+N_{5}+N_{6}-N_{7}-N_{8}-N_{9}+N_{10}\right. \\
& \left.+N_{11}+N_{12}-N_{15}-N_{14}-N_{15}+N_{16}\right) \\
& v_{12}=\frac{1}{\sqrt{2}}\left(N_{3}-N_{4}+N_{5}-N_{6}-N_{11}-N_{12}-N_{13}+N_{14}\right) \\
& " v_{15}-\frac{\mathrm{l}}{\sqrt{2}}\left(N_{1}-N_{2}-N_{7}+N_{8}-N_{0}+N_{16}+N_{15}-N_{16}\right) \\
& { }^{a} v_{14}=\frac{1}{2}\left(N_{1}+N_{2}-N_{3}-N_{4}-N_{5}-N_{6}+N_{7}+N_{8}-N_{9}-N_{111}\right. \\
& \left.+N_{11}+N_{12}+N_{15}+N_{14}-N_{15}-N_{16}\right) \\
& { }^{a} v_{15}=\frac{1}{\sqrt{2}}\left(N_{3}-N_{4}-N_{5}+N_{6}-N_{11}+N_{12}+N_{13}-N_{14}\right) \\
& { }^{a} v_{10}=N_{1}+N_{2}+N_{3}+N_{4}+N_{5}+N_{91}-N_{7}+N_{8}-N_{9}-N_{(6)} \\
& -N_{11}-N_{12}-N_{13}-N_{14}-N_{15}-N_{16} \equiv " v_{31}
\end{aligned}
$$
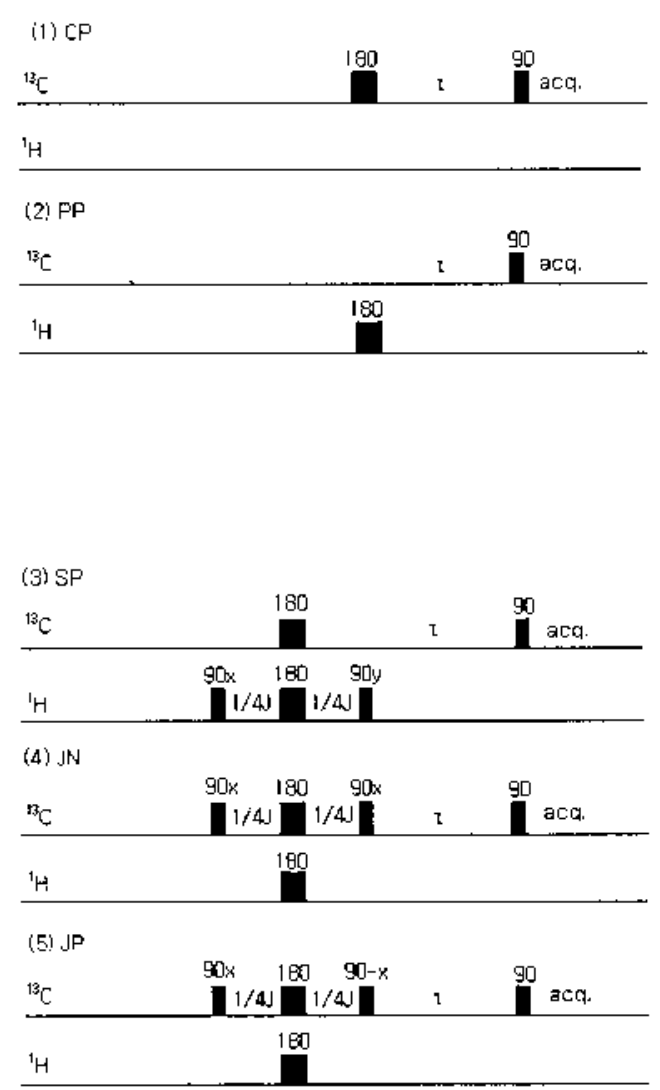

Fach of these magnetization modes will relax according to Eq. (4) for which the elements of relaxation matrix $\Gamma$ are given in Appendix 1. Among these, the modes of our interest are " $v_{1}$, the total $A$ magnetization. " $v_{2}$, the total $X$ magnetization, " $v$, the difference in integral between the sum of outer two lines and the inner single line of the broad $A$ triplet, " $v_{s}$, the difference in integral between the outer two lines of the broad unresolved $A$ triplet, and " $v_{i / \text {, the }}$ the tal $M$ magnetization. The modes " $v$, to " $v_{15}$ are all the $M$-related transition modes.

Among the $M$-related modes by far the most dominant is " $v_{11}$ mode, because this term remains much larger than the others during the relaxation. So, to a good approximation we may expect the effect of the presence of vicinal protons to be accounted for by considering only the cross relaxation between the $A X_{2}$ modes and " $v_{i}$ mode. Evaluation of the relaxation matrix for the weakly coupled $A V_{2} M$ brought the following facts to our attention. First, for the $A X_{2} M$ spin system diagonal and cross terms relating the modes (" $v_{1}$ to $\left.{ }^{\prime \prime} V_{7}\right)$ with each other are the same as those for the $A X_{2}$ spin system providing the dipolar terms involving $M$ spin are viewed as random field terms. Second, only " $v_{\text {. }}$. and " $v_{3}$ mode among " $v_{1}$ to " $v_{7}$ are influenced by the relaxation of " $v_{i /}$ mode. Third, all the cross relaxation elements between modes related to the transitions for the $A X_{2}$ spin system and those for $M$ spin involve cross spectral densities between $A Y_{2}$ spins and $M$ spin. These elements are expected to be
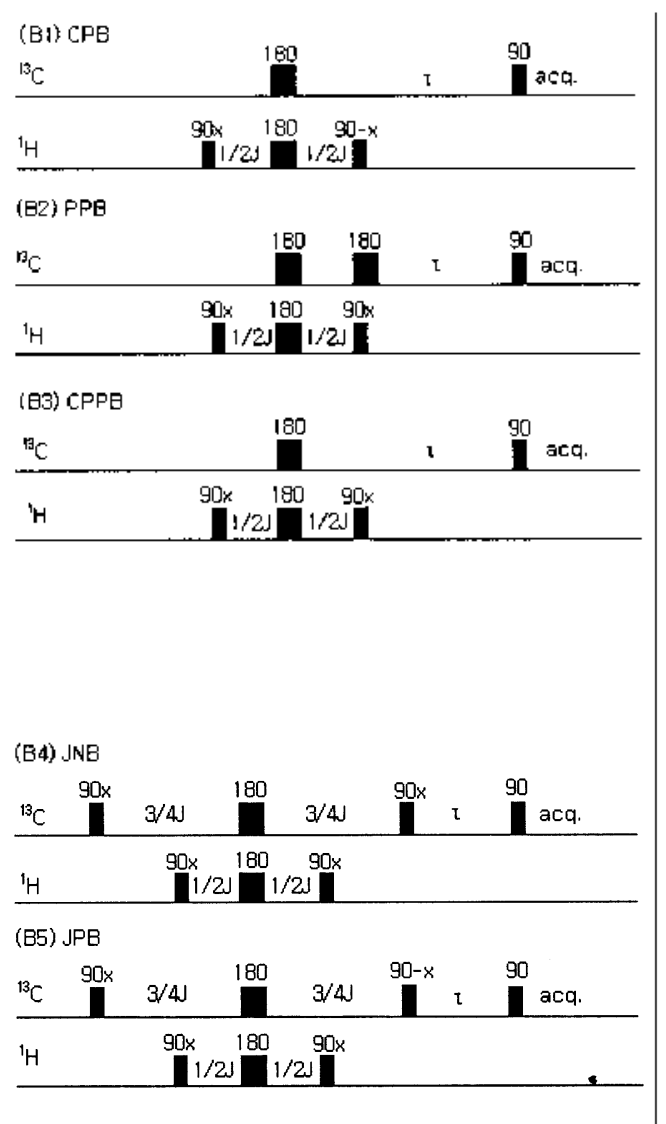

Figure 3. Pulse Secuences Used for Initial Vxcitation ol Observable Magyneti/ation Modes. 
sntall in magnitude considering that the presence of vicinal protons provides merely a small perturbation. Since the Grant-type pulse sequences and the BIRD-type pulse sequences both generate almost the same initial states of the $M$-related transition modes. ${ }^{a} V$ to ${ }^{a} V_{15}$, we can safely ignore the cross relaxation terns involving the $M$-related transition modes except ${ }^{a} v_{M}$. Based on this simplified treatment we could successfully fit the derived relaxation curves for various normal modes with the observed ones including those obtained by applying the BIRD-type pulses.

\section{Experimentals and Calculations}

Sample. The $\left[5 \cdot{ }^{13} \mathrm{C}\right] n$-undecane was synthesized in our laboratory. This was dissolved in $\mathrm{CDCl}_{3}$ and sealed in $5 \mathrm{~mm}$ NMR tubes after repeating five freeze-punip-thaw cycles to remove dissolved oxygen. The NMR experiments were performed on an Varian VXR 200S spectrometer operating at a ${ }^{13} \mathrm{C}$ frequency of $50.3 \mathrm{MHz}$. The temperature was maintained at $298 \mathrm{~K}$ throughout the measurements.

Pulses. The Grant pulse sequences invert both geminal and vicinal protons using a hard $180^{\circ}$ proton pulse while in the BIRD-type pulse sequences only the geminal protons in ${ }^{13} \mathrm{CH}_{2}$ moiety are inverted leaving the vicinal protons unaffected. The comparison between these two types of relaxation experiments is expected to reveal the effects of vicinal protons on the determination of the spectral densities.

In the present investigation the following five different Grant-type initial perturbations for the ${ }^{13} \mathrm{CH}_{3} \mathrm{M}$ spin system were used: (l) a ${ }^{13} \mathrm{C} \quad 180^{\circ}$ pulse inverting the entire methylene triplet (abbreviated to $\mathrm{CP}$ ). (2) a ${ }^{1} \mathrm{H} 180^{\circ}$ pulse inverting the proton doublet (denoted by PP for brevity), (3) selective ${ }^{1} \mathrm{H} 180^{\circ}$ pulse inverting the only the upfield line of the ${ }^{1} \mathrm{H}$ doublet (SP), (4) a $J$-negative pulse inverting the outer lines of the ${ }^{13} \mathrm{C}$ triplet $(J N)$, and $(5)$ a $J$-positive pulse inverting the central line of the ${ }^{13} \mathrm{C}$ triplet (JP). Besides these. we have applied the following five different BIRDtype initial perturbations for the ${ }^{13} \mathrm{CH}_{2} \mathrm{M}$ spin system: (1) a ${ }^{i 3} \mathrm{C} 180^{\circ}$ pulse inverting the entire methylene triplet and a ${ }^{1} \mathrm{H}$ $180^{\circ}$ pulse inverting the only neighboring protons (CPB). (2) a ${ }^{1} \mathrm{H} 180^{\circ}$ pulse inverting the only protons of ${ }^{13} \mathrm{CH}_{2}$ (PPB). (3) $\mathrm{a}^{13} \mathrm{C} 180^{\circ}$ pulse inverting the entire methy lene triplet and a ${ }^{l} \mathrm{H} 180^{\circ}$ pulse inverting the only protons of ${ }^{13} \mathrm{CH}_{2}$ (CPPB). (4) a $J$-negative pulse inverting the outer lines of the ${ }^{13} \mathrm{C}$ triplet and a ${ }^{1} \mathrm{H} 180^{\circ}$ pulse inverting the only protons of ${ }^{13} \mathrm{CH}_{2}(\mathrm{NNB})$. (5) a $J$-positive pulse inverting the central line of the ${ }^{13} \mathrm{C}$ triplet and a ${ }^{1} \mathrm{H} 180^{\circ}$ pulse inverting only the protons of ${ }^{13} \mathrm{CH}_{2}$ (JPB).

Calculations and Relaxation Curve Fittings. Relaxation parameters were obtained through a multiparameter leastsquares curve fitting of observed relaxation data with those derived from $\mathrm{Eq}$. (4). We have used the LevenbergMarquardt algorithm for searching the minima throughout these curve-fitting procedures. ${ }^{21.22}$ To account for systematic instrumental errors, differences in $T_{2}^{*}$ relaxation of each line during the pulse sequences. and pulse imperfections we parameterized the initial values of the normal modes. For
Table 1. Best Fitted Spectral Densities for $\left[5^{1}{ }^{13} \mathrm{C}\right] n$-Undecane

\begin{tabular}{|c|c|c|c|}
\hline & $\mathrm{AL}_{2}^{a}$ & $A \mathrm{I}_{2}^{\circ}$ & $A M_{2}$ \\
\hline$J_{\mathrm{CH}}$ & $0.0369 \pm 0.0008$ & $0.0347 \pm 0.0020$ & $0.0305 \pm 0.0003$ \\
\hline$J_{\mathrm{HCH}}$ & $0.0043 \pm 00036$ & $0.0041 \pm 0.0086$ & $0.0026 \pm 0.0021$ \\
\hline$J_{\mathrm{CHH}}$ & $0.0218 \pm 0.0010$ & $0.0210 \pm 0.0026$ & $0.0228 \pm 0.0004$ \\
\hline$J_{\mathrm{HH}}$ & $0.0335 \pm 0,0017$ & $0.0310 \pm 0.0048$ & $0.0447 \pm 0.0009$ \\
\hline$j c$ & {$[0.0]^{\mathrm{c}}$} & $0.0088 \pm 0.0091$ & {$[0.0114 \pm 0.0005]^{2 t}$} \\
\hline$j_{\mathrm{H}}$ & $0.0789 \pm 0.0034$ & $0.0808 \pm 0.0096$ & $0.0615 \pm 0.0016$ \\
\hline$j_{\mathrm{HH}}$ & $0.0500 \pm 0.0186$ & $0.0554 \pm 0.0445$ & $0.0163 \pm 0.0085$ \\
\hline$\Gamma_{116}$ & & & $0.0114 \pm 0.0005$ \\
\hline$\Gamma_{216}$ & & & $0.0354 \pm 0.0033$ \\
\hline$\Gamma_{1616}$ & & & $0.2787 \pm 0.0094$ \\
\hline$x^{2} / \mathrm{N}$ & $9.94 \times 10^{-5}$ & $1.03 \times 10^{-4}$ & $2.02 \times 10^{-1}$ \\
\hline
\end{tabular}

$A \mathrm{~F}_{2}$ denotes the results of the $\mathrm{Al}_{2}$ model fit of the relasation data obtained by applying the Grant type pulses only. ${ }^{b} A 141$ denotes the results of the $A 1 \mathrm{H} / 1$ model fit of the relaxation data obtained by both the Grant and BIRD-type pulses. "Square bracket denotes that the value was kept fixed during the fit. "Square bracket denotes that random field term for ${ }^{13} \mathrm{C}$ was assumed to originate only from dipolar term between ${ }^{13} \mathrm{C}$ and $1 /$ proton.

this, we first guessed a set of rough initial values for these normal modes and fitted the relaxation data using the relaxation matrix elements obtained in the case of $A X_{2}$ model, to find a more refined set of initial values. These initial values are then fed back to find a set of matrix elements that produces better curve fittings. This procedure was continued until the self-consistent best-fitted parameters were obtained. In particular the starting initial value for ${ }^{\text {a }} \mathrm{M}$ was set to -1 when a hard $180^{\circ}$ proton pulse was applied but to 0 when a BIRD-type selective $180^{\circ}$ proton pulse was applied.

\section{Results and Discussion}

To interpret the observed data we first tried to fit the relaxation data obtained by applying only the Grant-type pulse sequences with the relaxation curves derived on the basis of $A X_{2}$ model. This resulted in a good fit. yielding a set of spectral density values as shown in Table 1 (but not graphically shown). However, use of these spectral density values failed to reproduce the relaxation data obtained by applying the BIRD-type pulse sequences very well as shown also in Figure 4. which indicates that our methylene moiety is not so simple as to be satisfactorily described as a simple $A X_{2}$ system. In other words. this is to say that the effect of the vicinal protons on ${ }^{13} \mathrm{CH}_{2}$ relaxation cannot be approximated as arising from random field terms only.

In order to get over this hurdle we invoked the $A X_{-} M$ model that was described in the previous section, which yielded successful simultaneous fittings with both types of relaxation data as shown in Figure 5. In these calculations the modes $v_{9}$ through $v_{15}$ except ${ }^{a} v_{4}$ were ignored during the relaxation as we had reasoned in the previous section. Although we treat our system on the basis of an $A X M$ model, it is not a truly isolated $A X M$ system, however, because all the spins involved are more or less are interacting with other surrounding protons. In particular, the 

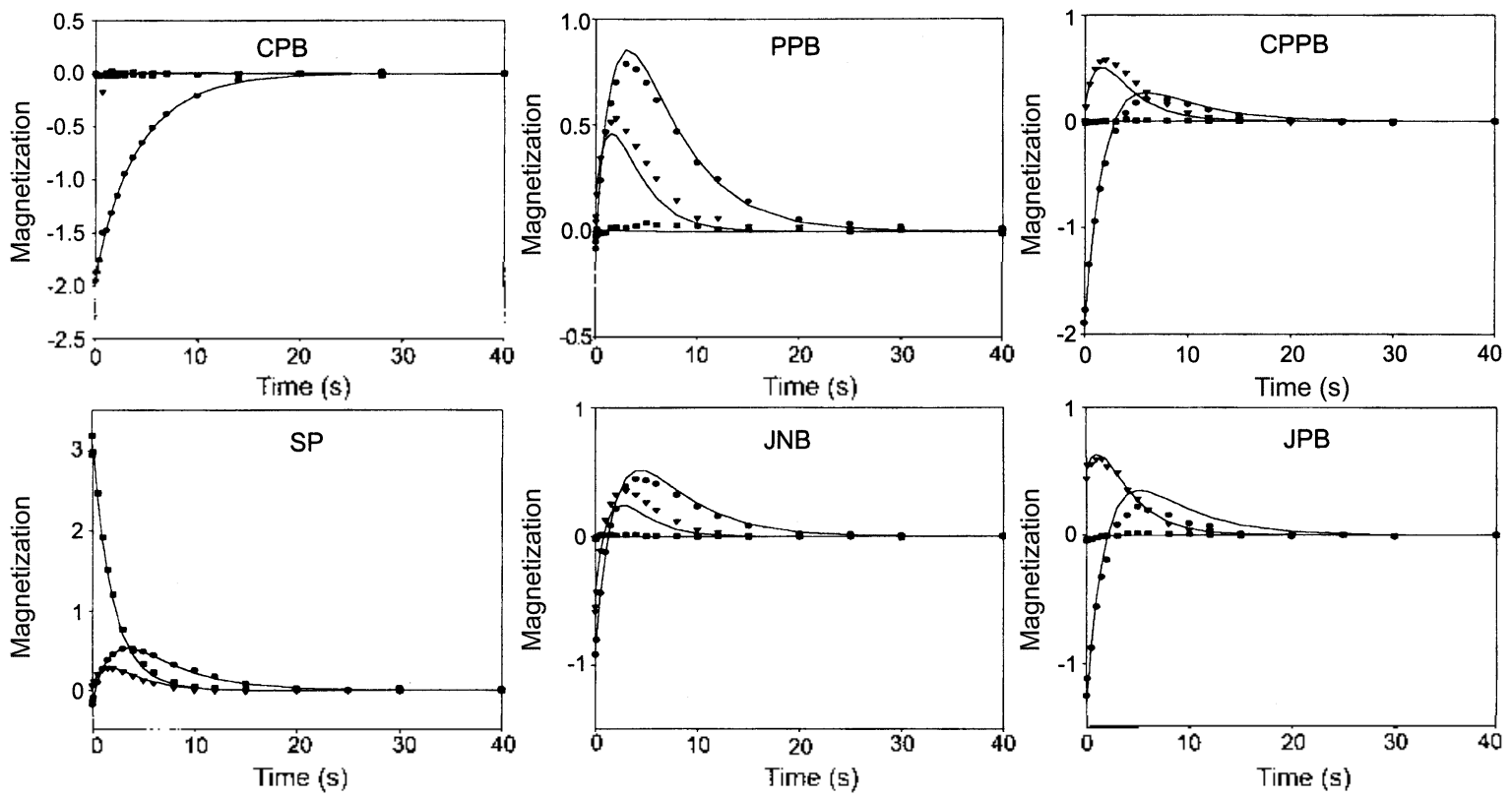

Figu re 4. Plot of Magnetization Modes Obtained from the Corresponding Coupled ${ }^{13} \mathrm{C}$ Spectra. The symbols denote the experimental data obtaincd by applying the BIRD type pulse sequences and solid lines are the curves fitted to these on the basis of the A.Y. relaxation matrix obtained from the Girant type experiments. 1 : $v_{1}$ mode, $\nabla:$ " $v_{3}$ mode, $\mathbf{a}: " v_{s}$ mode
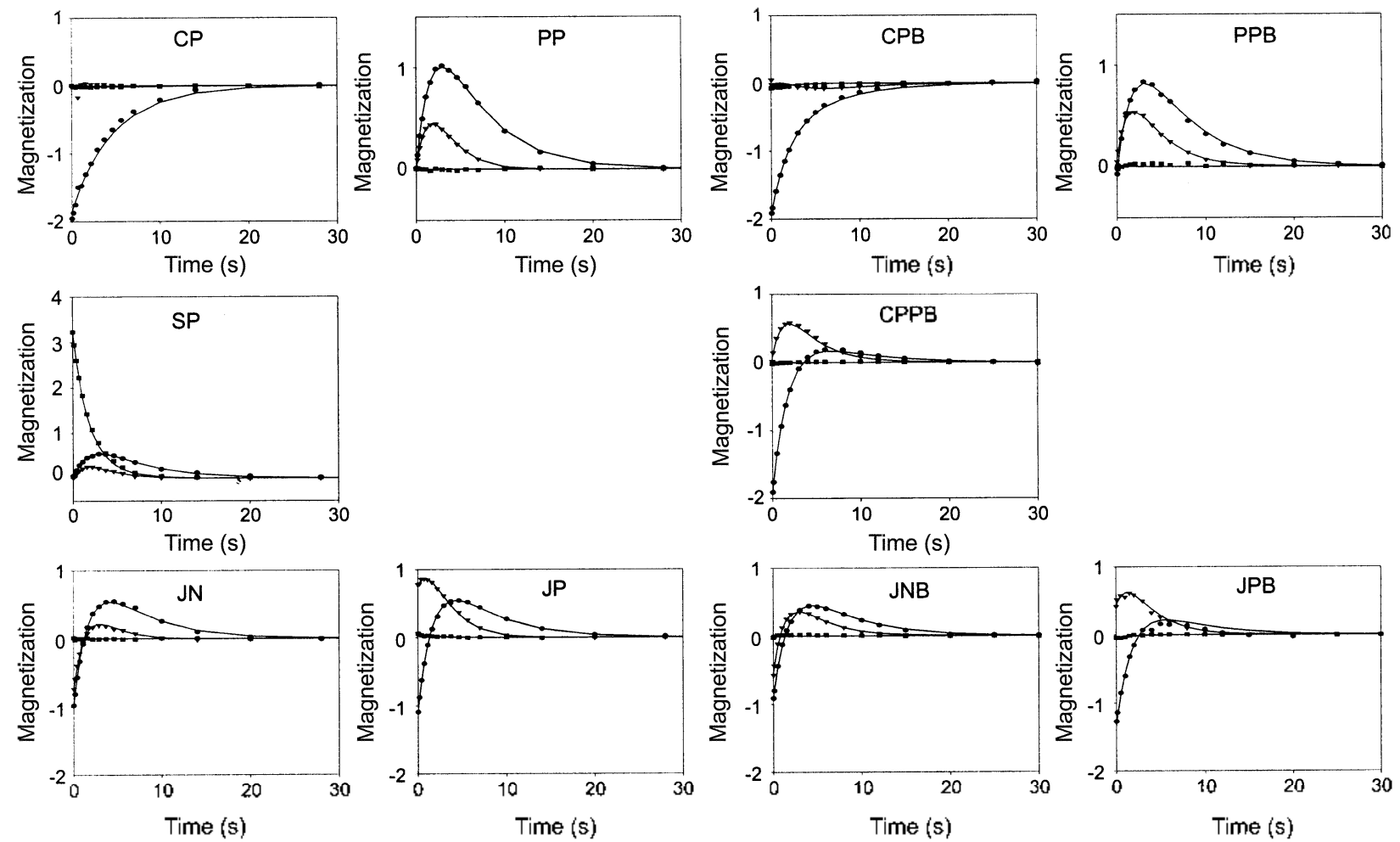

Figure 5. Plot of Magnetization Modes Obtained from Corresponding Coupled ${ }^{13} \mathrm{C}$. Spectra, lhe symbols denole the experimental data and solid lines represent the eurves fitted to these on the basis of $1 X_{2} \mathrm{H} / \mathrm{rel}$ axation model. $\bullet:$ " $v_{1}$ mode. $\mathbf{v} ; " v_{3}$ mode. $\mathbf{a}: " v_{5}$ mode

spin $M$ has another independent efficient relaxation pathway through interactions with the surrounding protons other than $A X_{2}$ spins. To account for this we have introduced an additional element $1 / T$ in the column of relaxation matrix that relates the "$v_{i r}$ mode with other modes. In the proton spectrum of our sample molecule $n$-undecane $M$ spin displays a somewhat broad, not well-resolved peak and this peak is found to decay nearly exponentially when inverted by a hard $180^{\circ}$ pulse, which enables us to estimate a crude value of $\%_{1}$. This value could be further refined through the curve fitting procedure as already described.

The spectral density values obtained through the least- 
squares curve fitting procedure are listed in Table 1 . We see from these that the various spectral density data calculated on the basis of the $A X_{2}$ model are affected substantially if the presence of vicinal protons is taken into consideration. The vicinal protons are expected to make their presence felt by the carbon of our interest wia direct dipole-dipole interactions as well as random field interactions. Although two groups of vicinal protons around the carbon labeled at the [5] position in undecane may be motionally inequivalent. we found that only two parameters are needed to describe satisfactorily the effect due to vicinal protons in our case. That is, we had $J_{\mathrm{CM}}=0.00171\left(5.6 \%\right.$ of $\left.J_{\mathrm{CH}}\right)$ and $J_{\mathrm{HMI}}=$ $0.00375\left(8.4 \%\right.$ of $\left.J_{\mathrm{HH}}\right)$ on the average for each of four vicinal protons. Our success may be due to the fact that the effect arising from the vicinal protons is merely secondary to that of the geminal protons. so that the sniall difference in $J_{\mathrm{CM}}$ and $J_{\mathrm{HM}}$ between the two groups of wicinal protons does not make significant contribution to the fitting results. These parameters are small in magnitude compared with $J_{\mathrm{CH}}$ and $J_{\mathrm{HH}}$. respectively. but large enough to indicate that the effect of vicinal protons cannot be fully described by random field interactions only. And all the spectral density values deduced from the fitting results differ from the case of $A X_{2}$ model by more than $5 \% .^{-33}$

One conventional method that is usually considered to minimize the effect due to vicinal protons is to deuterate them. However. this method is not easy to be applied for the type of experiments we described here. because not only the vicinal protons should be deuterated but at the same time the carbon of interest must be isotopically labeled as well for the sake of signal intensity. Fuson et al. have discussed the effect of vicinal protons on the relaxation of central carbon in $n$-nonane by comparing the measured data for deuterated and nondeuterated compound. They have also found that $J_{\mathrm{CH}}$ decreases only slightly in the presence of vicinal protons and have reconmended that $J_{C H}$ values calculated from the $A X_{2}$ model can be used for the study of molecular dynamics without much ado. However, the deuterated nonane molecule that they have used for comparison is a heavily deuterated species; i.e. the molecule in which all the protons. let alone the vicinal protons. except those in central methylene moiety of interest were deuterated. Such a heavily deuterated molecule may exhibit somewhat different dynanical behavior in comparison with a non-denterated species due to increased mass and moment of inertia. Therefore. it remains to be seen that deuteration is a sure way to eliminate the uncertainty in the values of various spectral densities. In this respect the method we propose in this paper may be considered to provide a viable way of serving for this purpose.

Acknowledgment. This research was financially supported by the BK2l project of the Ministry of Education. Korea.

\section{Appendices}

\section{Relaxation matrix elements for $A X=M$}

$\Gamma_{l l}=20 / 3 J_{\mathrm{CH}}+10 / 3 J_{\mathrm{CM}}+2 j C \quad \Gamma_{12}=5 \sqrt{2} / 3 J_{\mathrm{CH}}$

$$
\begin{aligned}
& \Gamma_{13}=2 J_{\mathrm{HCH}} \quad \Gamma_{14}=7 \sqrt{2} / 3 J_{\mathrm{HCH}} \\
& \Gamma_{113}=2 \sqrt{2} J_{\mathrm{HCM}} \quad \Gamma_{116}=5 / 3 J_{\mathrm{CM}} \\
& \Gamma_{22}=10 / 3 J_{\mathrm{CH}}+5 J_{\mathrm{HH}}+10 / 3 J_{\mathrm{HM}}+2 j_{\mathrm{H}} \\
& \Gamma_{23}=2 \sqrt{2} J_{\mathrm{CHH}} \quad \Gamma_{24}=5 / 3 J_{\mathrm{HCH}}-2 J_{\mathrm{CHH}} \quad \Gamma_{213}=2 J_{\mathrm{CHM}} \\
& \Gamma_{214}=2 \sqrt{2} J_{\mathrm{HHV}} \quad \Gamma_{115}=-2 J_{\mathrm{HHVI}}+5 / 3 J_{\mathrm{H} \backslash \mathrm{HH}} \\
& \Gamma_{216}=5 \sqrt{2} / 3 J_{\mathrm{HM}} \\
& \Gamma_{33}=4 J_{\mathrm{CH}}+10 / 3 J_{\mathrm{CM}}+2 J_{\mathrm{HH}}+20 / 3 J_{\mathrm{HM}}+2 j_{\mathrm{C}}+4 j_{\mathrm{H}} \\
& \Gamma_{34}=-\sqrt{2} J_{\mathrm{HCH}}-\sqrt{2} J_{\mathrm{HH}}-10 \sqrt{2} / 3 J_{\mathrm{HMH}}-2 \sqrt{2} j_{\mathrm{HH}} \\
& \Gamma_{313}=2 \sqrt{2} J_{\mathrm{HCM}}+2 \sqrt{2} J_{\mathrm{HHM}}+5 \sqrt{2} / 3 J_{\mathrm{HM}} \\
& \Gamma_{314}=4 J_{\mathrm{CHM}}+5 / 3 J_{\mathrm{CM}} \quad \Gamma_{315}=-2 \sqrt{2} J_{\mathrm{CHHM}} \\
& \Gamma_{44}=14 / 3 J_{\mathrm{CH}}-4 / 3 J_{\mathrm{HCH}}+10 / 3 J_{\mathrm{CM}}+J_{\mathrm{HH}}+20 / 3 J_{\mathrm{HM}} \\
& -10 / 3 j_{\mathrm{HVH}}+2 j_{\mathrm{C}}+4 j_{\mathrm{H}}-2 j_{\mathrm{HH}} \\
& \Gamma_{413}=-2 J_{\mathrm{HHM}}-5 / 3 J_{\mathrm{HMH}} \quad \Gamma_{414}=-2 \sqrt{2} J_{C \mathrm{HHM}} \\
& \Gamma_{415}=14 / 3 J_{C \mathrm{HM}}-8 / 3 J_{\mathrm{CHHM}}+5 / 3 J_{C \mathrm{M}} \\
& \Gamma_{5 S}=16 / 3 J_{\mathrm{CH}}+2 J_{\mathrm{HCH}}+10 / 3 J_{\mathrm{CM}}+5 J_{\mathrm{HH}}+10 / 3 J_{\mathrm{HM}}+2 j_{\mathrm{C}} \\
& +2 j_{\mathrm{H}} \\
& \Gamma_{56}=5 \sqrt{2} / 3 J_{C \mathrm{H}}+2 \sqrt{2} J_{C \mathrm{HH}} \quad \Gamma_{57}=-5 / 3 J_{\mathrm{HCH}}-2 J_{C \mathrm{HH}} \\
& \Gamma_{59}=2 \sqrt{2} J_{\mathrm{HCM}}+5 \sqrt{2} / 3 J_{\mathrm{HM}} \Gamma_{51 \mathrm{j}}=2 J_{\mathrm{CHM}}+5 / 3 J_{\mathrm{CM}} \\
& \Gamma_{511}=2 \sqrt{2} J_{\mathrm{HCM}}+2 \sqrt{2} J_{\mathrm{HHM}} \quad \Gamma_{5 \mathrm{l}}=-2 J_{\mathrm{HHM}}+5 / 3 J_{\mathrm{HMH}} \\
& \Gamma_{66}=20 / 3 J_{\mathrm{CH}}+2 J_{\mathrm{HH}}+20 / 3 J_{\mathrm{HM}}+4 j_{\mathrm{H}} \\
& \Gamma_{67}=-10 \sqrt{2} / 3 J_{\mathrm{HCH}}-\sqrt{2} J_{\mathrm{HH}}-10 \sqrt{2} / 3 J_{\mathrm{HMH}}-2 \sqrt{2} j_{\mathrm{HH}} \\
& \Gamma_{6 l l}=2 \sqrt{2} J_{\mathrm{HHM}}+5 \sqrt{2} / 3 J_{\mathrm{HM}} \quad \Gamma_{6 l l}=4 J_{\mathrm{CHM}} \\
& \Gamma_{612}=-2 \sqrt{2} J_{C \mathrm{HHM}} \\
& \Gamma_{77}=20 / 3 J_{\mathrm{CH}}-10 / 3 J_{\mathrm{HCH}}+J_{\mathrm{HH}}+20 / 3 J_{\mathrm{HM}}-10 / 3 J_{\mathrm{HMH}}+4 j_{\mathrm{H}} \\
& -2 j_{\mathrm{HH}} \\
& \Gamma_{710}=-2 J_{\mathrm{HHM}}-5 / 3 J_{\mathrm{HMM}} \quad \Gamma_{7] 1}=-2 \sqrt{2} J_{C \mathrm{HHM}} \\
& \Gamma_{712}=14 / 3 J_{\mathrm{CHM}}-8 / 3 J_{\mathrm{CHHM}} \\
& \Gamma_{99}=20 / 3 J_{C \mathrm{H}}+2 J_{\mathrm{CM}}+20 / 3 J_{\mathrm{HM}}+2 j_{C}+2 j_{\mathrm{M}} \\
& \Gamma_{91 \mathrm{l}}=5 \sqrt{2} / 3 J_{\mathrm{CH}}+2 \sqrt{2} J_{\mathrm{CXH}} \quad \Gamma_{9 l 1}=2 J_{\mathrm{HCH}}+2 J_{\mathrm{HNM}} \\
& \Gamma_{912}=7 \sqrt{2} / 3 J_{\mathrm{HCH}}+7 \sqrt{2} / 3 J_{\mathrm{HMH}} \\
& \Gamma_{[!] ! !}=10 / 3 J_{C \mathrm{H}}+10 / 3 J_{C \mathrm{M}}+5 J_{\mathrm{HH}}+16 / 3 J_{\mathrm{HM}}+2 J_{\mathrm{HMH}}+2 j_{\mathrm{H}} \\
& +2 \mathrm{j}_{\mathrm{M}} \\
& \Gamma_{1 \dot{1 j 1} 1}=2 \sqrt{2} J_{\mathrm{CHH}}+2 \sqrt{2} J_{\mathrm{CMH}} \quad \Gamma_{1 \dot{1012}}=5 / 3 J_{\mathrm{HCH}}-2 J_{\mathrm{CHH}} \\
& \Gamma_{1111}=4 J_{\mathrm{CH}}+2 J_{\mathrm{CM}}+2 J_{\mathrm{HH}}+4 J_{\mathrm{HM}}+2 j_{\mathrm{C}}+4 j_{\mathrm{H}}+2 j_{\mathrm{M}} \\
& \Gamma_{1112}=-\sqrt{2} J_{\mathrm{HCH}}-\sqrt{2} J_{\mathrm{HH}}-\sqrt{2} J_{\mathrm{HMH}}-2 \sqrt{2} j_{\mathrm{HH}} \\
& \Gamma_{1312}=14 / 3 J_{\mathrm{CH}}-4 / 3 J_{\mathrm{HCH}}+2 J_{\mathrm{CM}}+J_{\mathrm{HH}}+14 / 3 J_{\mathrm{HM}}-4 / 3 J_{\mathrm{H} M H} \\
& +2 j \mathrm{c}+4 j_{\mathrm{H}}-2 j_{\mathrm{HH}}+2 j \mathrm{M} \\
& \Gamma_{1313}=16 / 3 J_{\mathrm{CH}}+2 J_{\mathrm{HCH}}+2 J_{\mathrm{CM}}+5 J_{\mathrm{HH}}+16 / 3 J_{\mathrm{HM}}+2 J_{\mathrm{HVMH}} \\
& +2 j \mathrm{C}+2 j_{\mathrm{H}}+2 j_{\mathrm{M}} \\
& \Gamma_{13]_{4}}=5 \sqrt{2} / 3 J_{\mathrm{CH}}+2 \sqrt{2} J_{\mathrm{CHH}}+2 \sqrt{2} J_{\mathrm{CMH}} \\
& \Gamma_{1315}=-5 / 3 J_{\mathrm{HCH}}-2 J_{\mathrm{CHH}} \\
& \Gamma_{1316}=2 \sqrt{2} J_{\mathrm{CWH}}
\end{aligned}
$$




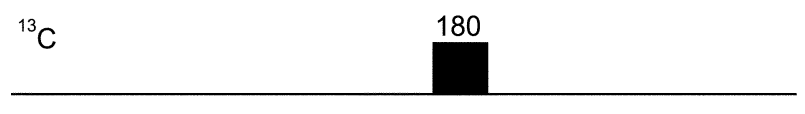

${ }^{1} \mathrm{H}$

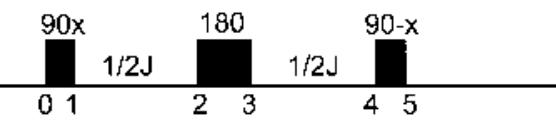

Figure A l. A BIRD Pulse Sequence.

$$
\begin{aligned}
& \Gamma_{\mid 414}-20 / 3 . J_{C+1}\left|10 / 3 . J_{\mathrm{CU}}\right| 2 J_{\mathrm{HH|}} \mid 4 J_{\mathrm{E| \textrm {N }}} \cdot 4 j_{\mathrm{H} \mid} \cdot 2 j_{\mathrm{M}}
\end{aligned}
$$

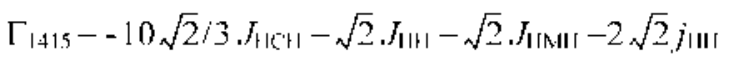

$$
\begin{aligned}
& \Gamma_{\mid 41 \text { r }}-2 J_{|| \mathrm{MII} \mid} \\
& \Gamma_{1515}-20 / 3 . J_{C \mathrm{H}}-10 / 3 . J_{\mathrm{HCH}} \text { । } 10 / 3 . J_{\mathrm{CN}}+J_{\mathrm{HH}} \cdot 14 / 3 . J_{\mathrm{HN}} \\
& -4 / 3 . J_{\mathrm{HMH}}+4 j_{\mathrm{H}}-2 j_{\mathrm{HH}}+2 j \mathrm{~N} \quad \Gamma_{1510}-7 \sqrt{2} / 3 . J_{\mathrm{HMH}} \\
& \Gamma_{\mid 616 \mathrm{r}}-10 / 3 J_{C \mathrm{CN}}+20 / 3 . J_{\mid \mathrm{XN}}+2 j \mathrm{~N}
\end{aligned}
$$

\section{Action of BIRD Pulse on $A X_{2} Y$ Spin System}

We analyze here the action of the BIRD-type pulses shown in Figure $\mathrm{Al}$ on an $A X_{2} Y$ spin system in which the spin coupling between $A$ and $X$ spins is much larger than both the spin coupling and the chemical shift difference between $X$ and $Y$ protons as well as the spin coupling between $A$ and $Y$ spin.

At equilibrium and after the first $90^{\circ}$ proton pulse, the density operators are, respectively, written as

$$
\begin{gathered}
\sigma_{0}=\alpha I_{z}-S_{1 z}+S_{2 z}+T_{z}, \\
\sigma_{1}-\alpha I_{z}-S_{1 y}-S_{2 y}-T_{y},
\end{gathered}
$$

where $I, S$ and $T$ are the angular momentum operators for carbon-13, geminat protons and a vicinal proton and $\alpha$ is $\gamma_{1} \gamma_{1}$ with $\gamma$ standing for the magnetogyric ratio of a nucleus of the given kind. After a BIRD pulse sequence, the density operator becomes

$$
\sigma_{5}-P(\pi / 2)_{x} L C(\pi) P(\pi)_{x} L \sigma_{1} L^{+} P(\pi)_{x}^{+} C^{\top}(\pi)^{-} L^{-} P(\pi / 2)_{x}^{-} .
$$

where $P(\pi / 2)_{x}, P(\pi)_{x}$, and $C(\pi)$ are, respectively, the operators describing the action of hard proton and carbon pulses; that is.

$$
\begin{aligned}
& \left.P(\pi / 2)_{r}-\exp \mid-i(\pi / 2)\left(S_{\mid r}+S_{2 v}+T_{x}\right)\right] . \\
& P(\pi)_{x}=\exp \left[-i \pi\left(S_{1 x}-S_{2 x}+T_{x}\right)\right], \text { and } \\
& C^{\top}(\pi)_{v}-\exp \left(-i \pi I_{r}\right) .
\end{aligned}
$$

$L$ is the operator for free evolution between two successive pulses; that is,

$$
L-\exp (-i H \tau)
$$

where $I /$ is the spin Hamiltonian for the given spin system and $\tau$ is the evolution period between hard proton $90^{\circ}$ and $180^{\circ}$ pulse. The spin Hamiltonian for the system may be written in the form

$$
H-H_{\omega}-H_{J},
$$

where $I_{t,}$ and $I_{f}$ are, respectively, the chemical shift part and the spin coupling part of the Hamiltonian expressed in unit of $\hbar$; that is,

$$
H_{v}-\omega_{y} I_{z}+\omega_{Y}\left(S_{1:}+S_{z-}\right)+\omega_{1} T_{z}
$$

and

$$
\begin{gathered}
H_{l}=\pi J \cdot 2 I_{z}\left(S_{12}+S_{2 s}\right)-\pi J^{\prime} \cdot 2 I_{I} T_{z} \\
+\pi J^{\prime \prime} \cdot 2\left(\mathbf{S}_{1}-\mathbf{S}_{2}\right) \cdot \mathbf{T}
\end{gathered}
$$

with the following definitions:

$\omega_{-4}=$ chemical shift frequency of carbon,

$\omega_{x}=$ chemical shift frequency of geminal protons,

$\omega_{r}=$ chemical shift frequency of vicinal proton.

$J=$ spin coupling constant between carbon and geminal protons.

$J^{\prime}=$ spin coupling constant between carbon and vicinal proton, and

$J^{\prime \prime}=$ spin coupling constant between geminal and vicind protons.

On action of simultaneous hard proton and carbon $180^{\circ}$ pulses the coupling part $H_{\text {, }}$ remains invariant while the chemical shift part changes its sign, and, therefore, we may rewrite (A3) as

$$
\begin{gathered}
\sigma_{5}=P(\pi / 2)_{x} L L^{\prime} C(\pi) P(3 \pi / 2)_{x} \sigma_{1} P(3 \pi / 2)_{x}^{\prime} \\
C(\pi)^{+}\left(L L^{\prime}\right)^{-} P(\pi / 2)_{r}^{-}
\end{gathered}
$$

where

$$
\left.L L^{\prime}-\operatorname{expl}-i\left(H_{0,}+H_{3}\right) \tau \mid \operatorname{expl}-i\left(-H_{\theta}+H_{3}\right) \tau\right]
$$

Since $H_{w}$ does not commute with $H_{j}$, two exponent operators in (A10) do not commute either and it is troublesome to deal with the operator $I L L^{\prime}$. However, we can deal with this operator approximately using the following formula: ${ }^{2-4}$

$$
\begin{gathered}
\exp (-i M \tau) \exp (-i N \tau)- \\
\exp \left[-i(M+N) \tau-\frac{\tau^{2}}{2}[M, N]-i \frac{\tau^{3}}{6}[M,|M, N|]+\cdots\right] .
\end{gathered}
$$

where $M^{-}-H_{i v}+H_{J}$ and $N-H_{i v}-H_{\gamma}$.

For $\tau-1 / 2 J$ the magnitude of the second term in the exponent series in (All) can be shown to be of the order of $\left(\Delta \omega J^{\prime \prime} / J\right)^{2}$ where $\Delta \omega$ is the chemical shift difference between vicinal and geminal protons. For our system $J$ is much larger than both $\Delta \omega$ and $f^{\prime \prime}$ and therefore the second and ensuing terms may safely be ignored. This amounts to writing

$$
I H^{\prime}=\exp \left(-2 i I_{,} \tau\right)
$$

$H_{j}$ operator itself still consists of two non-commuting parts, but by the same logic as we have used for deriving (AI2) we can show that

$$
\begin{gathered}
L L^{\prime} \approx \exp \left[-4 \pi J_{z}\left(S_{I_{I}}+S_{z z}\right) \tau\right] \exp \left(-4 \pi i J^{\prime} I_{z} T_{z} \tau\right) \\
\left.\operatorname{expl}-4 \pi i J^{\prime \prime}\left(\mathbf{S}_{1}+\mathbf{S}_{2}\right) \cdot \mathbf{T} \tau\right]
\end{gathered}
$$

On substituting (A13) into (A9), we see that for $\tau=1 / 2 J$ the carbon signal associated with proton zero-cjuantum coherence involving geminal and vicinal protons can arise if the non- 
secular part of the spin coupling between these two protons cannot be ignored and its intensity is roughly proportional to $\sin \left(2 \pi J^{\prime} / J\right) \sin \left(2 \pi J^{\prime \prime} / J\right)$ which is very sntall if $J$ is very large compared with both $J^{\prime}$ and $J^{\prime \prime}$.

\section{References}

1. Courtiel, I.: Gonord. P; Mavne, C. L. J. Chem. Phys. $1980,72$. 953.

2. Fuson. M. M.: Prestegard. J. H. J. Am. Chem. Soc. 1983. 105.168.

3. Fuson. M. M.: Prestegard. J. H. Biochemistry 1983. 22. 1311.

4. Brown, M. S.: Grant D. M.: Horton. W. I.: Mayne C. L.: Evans. G. T. J. Am. Chem. Soc 1985 107,6698.

5. Fuson. M. M.: Grant. D. M. Hacromolectles 1988, 21.944.

6. Fuson. M. M.: Grant. D. M. Macromolecules 1991. 24. 2594

7. Grant. D. M.: Mayne. C. L.: Liu. F.: Xiang. T.-X. Chent. Rev 1991. 91. 1591

8. Zheng. Z.: Mayne. C. L.; Grant. D. M. J. Magm. Reson. A 1993. 103,268

9. Xiang. T.-X: Liu, F.: Grant. D. M. J. Chem. Plns. 1991. $95,7576$.
10. Liu. F.: Horton. W. J.; Mavne C. L.; Xiang, T.-X.: Grant. D. M. $d$. Ant Chent. Soc. 1992. H1.5281.

11. Fuson1. M. M.: Belı A. M. J. Magn. Reson. 1994. J07. I.

12. Liu. F.: Mayne. C. L.: Grant. D. M. J. Magn. Resont 1989. 84. 344.

13. Vax. A. J. Hagni. Reson. $1983,53,517$.

14. Redfield, A. G. IBM IJ. Res. Develop. 1957, 1. 19.

15. Redfield. A. G. Adh Magn. Reson. 1965. l. 1.

16. Solomon1, I. Phws. Rev: 1955. 99. 559.

17. Wang. C. H.: Grant. D. M. J. Chem. Phys. 1976. 6t. 1522.

18. Pyper. N. C. Hol Plys. 1971. 21, 1.

19. Pyper. N. C. Hol. Plys. 1971. 22, 433

20. Werbelow, L. G.; Grant, D. M. Adn. Magn. Reson. 1977, 9. 189.

21. Bevingtonl. P. R. Data Reduction and Error Analysis for the Phisical Sciences: McGraw-Hill: 1969.

22. Press. W. H.: Flannery. B. P.: Teukolsy. S. A.: Vetterling. W. T. Numerical Recipes-The Art of Scientific Computing. Cambridge University Press: 1986.

23. Namgoong, H.: Lee, I.: Lee, J. W. Bull Korean Chem Soc 2000. 21. 1077 .

24. Goldberger. M. L.: Adams II. E. N. J. Chem. Phns. 1952. 20. 240. 